\title{
A novel ML DOA estimation algorithm for array signal processing
}

\author{
Pengfei Shi ${ }^{1}$, Xinnan $\mathrm{Fan}^{1, \mathrm{a}}$, Jianjun $\mathrm{Ni}^{1}$ and Dewei Yang ${ }^{2}$ \\ ${ }^{1}$ College of IOT Engineering, Hohai University, 213022 Changzhou, China \\ ${ }^{2}$ Nanjing Hydraulic Research Institute, Nanjing, 210029 China
}

\begin{abstract}
A novel algorithm for finding the optimal solution of nonlinear function in maximum likelihood DOA estimation is proposed to reduce the calculation in multi-dimensional nonlinear search of the estimation. In the proposed method, firstly, the mode of population initialization mode is modified to improve the stability of population evolution. Secondly, the crossover operator is also improved to enlarge the range of new generated individual. Thirdly, parameters adaptive adjustment strategy is designed to accelerate convergence. The simulation shows that the proposed algorithm can greatly reduce the calculation time.
\end{abstract}

\section{Introduction}

Direction of arrival (DOA) estimation is an important process in array signal processing[1]. The DOA estimation algorithms are mainly divided into three types[2]: conventional beamforming (CBF), subspacebased(SB) and maximum likelihood (ML). However, $\mathrm{CBF}$ is limited by the array physical aperture. It cannot distinguish between two spatial targets within a beam width[3]. SB cannot solve the computational complexity problem well when the number of sensors is large[4]. ML DOA estimation algorithm is a representative and practical estimation algorithm of DOA estimation theory. It can achieve a good estimation performance, especially in asymptotic estimation[5]. The ML DOA estimation has attracted more and more attention from researchers.

However, the ML DOA estimation contains a multidimensional nonlinear function, which will lead to a high computational cost. Researchers attempted to use intelligent algorithms based on bionics to solve the multidimensional nonlinear searching problem in ML DOA estimation. Such as the differential evolution (DE) algorithm[6], the artificial bee colony (ABC) algorithm[7], and the particle swarm optimization (PSO) algorithm[8].

In this paper, a fast algorithm of ML DOA estimation based on bee evolutionary genetic algorithm (BEGA) is proposed to optimize ML DOA estimation. BEGA introduces some excellent evolutionism of bee to emphasize the importance of the optimum individual in population evolution. It can converge to global optimal solution quickly[9].

The proposed algorithm mainly improves BEGA from three parts: population initialization, crossover operator and adaptive adjustment of parameters. Simulation results show that the algorithm not only keeps the estimated accuracy of original ML DOA estimation algorithm, but also significantly simplifies the calculation.

\section{The proposed algorithm}

\subsection{Signal Model of Array}

Assuming that a uniform linear array is made up of $\mathrm{M}$ elements and its element gap is d. The sketch of array receiving signal from far field source is as shown in Figure 1.

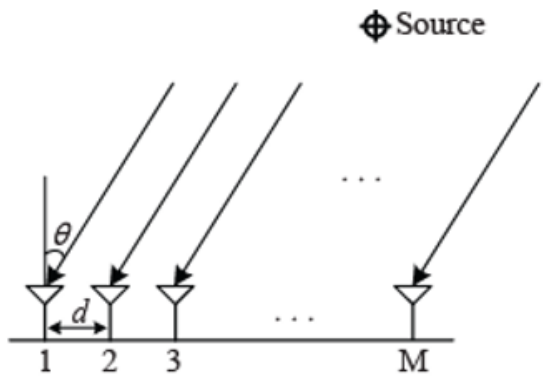

Figure 1. Sketch of array receiving signal

The ML estimation about parameter $\theta$ is calculated as Equation (1).

$$
\hat{\Theta}=\min _{\theta}\left\{\operatorname{tr}\left\{\mathbf{P}_{A}^{\perp} \hat{\mathbf{R}}\right\}\right\}=\max _{\theta}\left\{\operatorname{tr}\left\{\mathbf{P}_{A} \hat{\mathbf{R}}\right\}\right\}
$$

Where $\Theta=\left[\begin{array}{llll}\theta_{1} & \theta_{2} & \cdots & \theta_{P}\end{array}\right]^{T}, \mathbf{P}_{\mathbf{A}}$ is projection of matrix $\mathbf{A}, \operatorname{tr}\{\bullet\}$ is trace of a matrix, $\mathbf{P}_{A}^{\perp}$ is orthogonal projection matrix of matrix $\mathbf{A}$ and $\mathbf{A}^{+}$is pseudo-inverse of matrix A.

\footnotetext{
a Corresponding author: fanxn@hhuc.edu.cn
} 


\subsection{Algorithm Flow}

Figure 2 shows the flow of proposed algorithm. Firstly, it calculates sample covariance matrix of array received data. Then it carries out the ML DOA estimation with sample covariance matrix. In order to speed up the search of nonlinear global optimum solution while doing ML DOA estimation, this paper optimizes the search with the aid of improved BEGA. According to the character of ML DOA estimation, the algorithm is improved in three parts: population initialization method, crossover operator and adaptive adjustment of parameters.

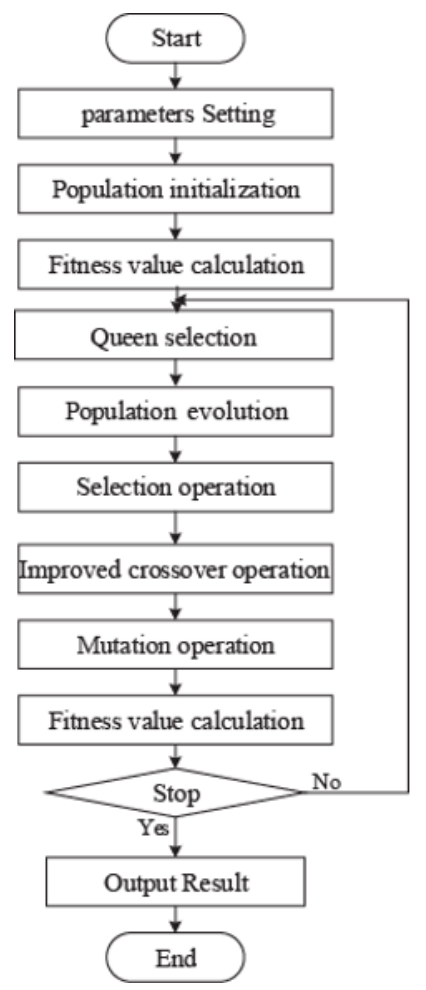

Figure 2. A fast algorithm for ML DOA Estimation

\subsection{Population Initialization}

Population initialization is the first step for population evolution and it provides an initial guess. Then, these initially guessed solutions will be iteratively improved in the course of the optimization process until a stopping criterion is met[10]. Generally, population initialization can affect the convergence speed and also the quality of the final solution[11]. Good initial population facilitates a GA's convergence to good solutions while poor initial population can hinder the convergence.

The random initialization is a default method of population generation. It employs pseudo-random number generator(PRNG) to produce the initial population[12]. The rationale behind this is that the PRNG can generate uniformly distributed samples and thus a population initialized using PRNG tends to cover promising regions which contains global optima or good local optima of the search space. Since the population size is always limited, the chance for a population to cover promising regions of the search space decreases as increasing the dimension of the search space. So, increasing the chance for a population to cover promising region of the search space is important.

Thus, in order to improve the traditional random initialization, solution space is divided into some subspace, and then the PRNG is used to get random result in each subspace as the initial population. It can be expressed with Equation (2).

$$
p[i]=[r(0,1)+(i-1)] \times \frac{180^{*}}{l}+(-90), i=1, \ldots, l
$$

Where $\mathrm{r}(0,1)$ is random number in interval $[0,1], \mathrm{p}[\mathrm{i}]$ is the ith individual of initial population and 1 is the size of population.

\subsection{Crossover Operator}

In BEGA, the optimum individual is chosen as the queen to operate the crossover with all the individuals in population. The range of new individual obtained by crossover operator represents the excavation ability of the crossover operator does with the optimum individual. Thus, this paper utilizes the improved crossover operator to expand the range of new individual to both sides of the queen. First, the crossover range is expanded to $[Q-\Delta, Q+\Delta]$, and then PRNG is used to achieve crossover result. It can take fully advantage of the information in queen. Advanced crossover operator can be expressed with Equation (3).

$$
p_{-} n[i]=Q+r(-1,1) \times\left|Q-p_{-} s[i]\right|
$$

Where $Q$ is the queen in each generation, $p \_n[i]$ is ith new individual obtained after crossover, p_s[i] is ith individual selected to crossover and $\mathrm{r}(-1,1)$ is random number in interval $[-1,1]$.

\subsection{Adaptive Adjustment of Parameters}

The performance of BEGA is determined by the balance between depth and breadth search in searching space. Adjusting the parameters to keep the search breadth as large as possible with the precision of solution meeting the requirement. Parameters affect evolution of BEGA are mutation probability $\mathrm{Pm}$, random individual range $\mathrm{Rs}$ and mutation interrupt range $\mathrm{Rm}$. Pm is used to control the probability of mutation occurrence. $\mathrm{Rm}$ is used to control the range of mutation, means the range of individual $x$ after mutation is $[x-R m, x+R m]$. Mutation operation is an auxiliary method of new individual generations and it determines ability of local search.

For ML DOA estimation, mutation operation not only controls the search range, but also determines the search precision. Increasing Pm and reducing $\mathrm{Rm}$ can shrink local search range and increase the depth of local search. It means that algorithm can obtain more possible solutions in unit range. Rs is used to control the range of random individual generate around near optimal solution. Increasing Rs can keep the waiting population at a high diversity. Thus it can avoid losing useful genetic 
information and improve the global convergence of algorithm.

In order to balance depth and breadth search and obtain the co-evolution of parameters and population, this paper adopts adaptive adjustment strategy as follows:

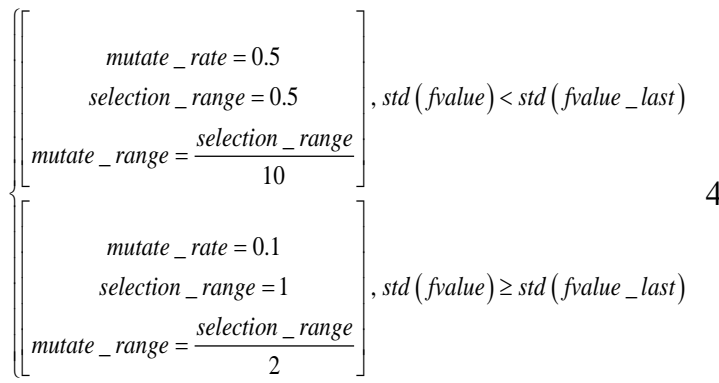

Where fvalue is fitness value sets of current population, fvalue_last is fitness value sets of previous population and $s t d(\bullet)$ is standard deviation. Standard deviation value reflects the discreteness of datasets. If fitness value sets meet $\operatorname{std}($ fvalue $)<$ std (fvalue_last $)$, it means that the evolution has a convergence trend. At the same time, increasing Pm and decreasing Rs and Rm can enhance the impact of local search in population evolution and expand the depth of local search. It is aimed to excavate high precision solution in the convergence direction of population evolution. Instead, $s t d($ fvalue $) \geq \operatorname{std}($ fvalue_last $)$ means that the evolution has a divergency trend. Decreasing Pm and increasing Rs and $\mathrm{Rm}$ can slack impact of local search and expand the breadth of global search. It can make algorithm jump out and avoid convergence to near optimal solution.

\section{Simulation and Analysis}

In this section, we demonstrate the simulation results of the proposed algorithm(PRO). Then we compare the proposed method with ABC, DE, and PSO search methods. For the sample data, the receiver array is supposed to be a 16 sensor uniform linear array and 500 data snapshots are used. Convergence rate and speed are important factors to evaluate the efficiency of an algorithm. The setting parameters of the proposed algorithm are shown in Table 1.

Table 1. Setting parameters of proposed algorithm

\begin{tabular}{|c|c|c|}
\hline Parameters & Value & Remarks \\
\hline 1 & 10 & population size \\
\hline $\mathrm{G}_{\max }$ & 500 & maximum generation \\
\hline $\mathrm{Pm}$ & 0.01 & mutation probability \\
\hline $\mathrm{Rs}$ & 1.0 & random individual range \\
\hline
\end{tabular}

\begin{tabular}{|c|c|c|}
\hline $\mathrm{Rm}$ & 0.5 & mutation interrupt range \\
\hline
\end{tabular}

In order to demonstrate the accuracy and reliability, the estimation process curves are obtained over an average of 500 times of the experiment. The curves are the estimation value found by the algorithm for each iteration versus the increasing number of iteration.

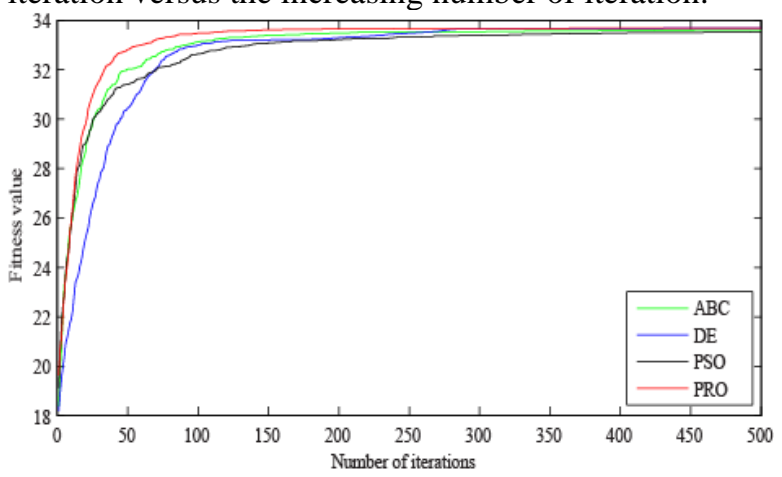

(a) $\mathrm{SNR}=0 \mathrm{~dB}$

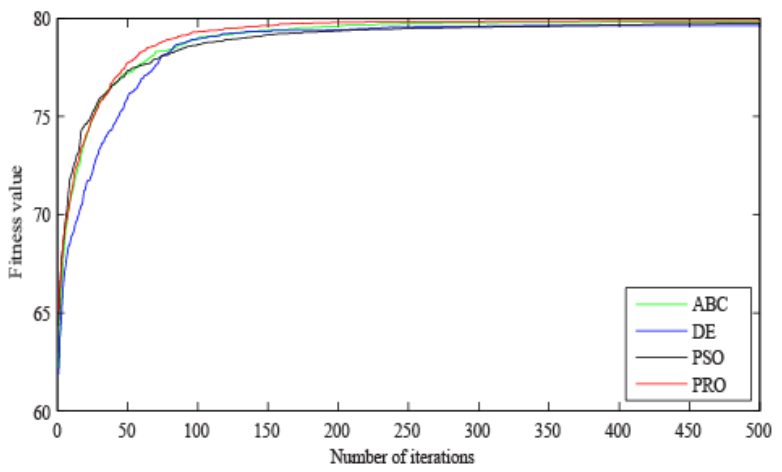

(b) $\mathrm{SNR}=-10 \mathrm{~dB}$

Figure 3. Fitness values of DE, ABC, PSO and PRO with two signal sources

Figure 3 shows the convergence properties of DE[6], $\mathrm{ABC}$ [7], $\mathrm{PSO}[8]$ and proposed algorithm with $\mathrm{SNR}=$ $0 \mathrm{~dB}$ and $\mathrm{SNR}=-10 \mathrm{~dB}$ respectively. As we see in the figure, the proposed algorithm reaches the final value faster than other algorithms no matter $\mathrm{SNR}=0 \mathrm{~dB}$ or $\mathrm{SNR}=-10 \mathrm{~dB}$. It means that the proposed algorithm can always converge to the optimal solution with fewer steps than other algorithms, which influence the computational load of the algorithm.

\section{Conclusion}

This paper improves the BEGA to optimize ML DOA estimation which can be used in the large amount of calculation problem of the nonlinear global search in ML DOA estimation. In the proposed method, the population initialization is modified to improve the stability of population evolution and the crossover operator is also improved to enlarge the range of new generated individual. At last, parameters adaptive adjustment strategy is designed to accelerate the convergence. All the improvements are verified to be effective according to simulation results. The simulation shows that the proposed algorithm can reduce the calculation time greatly. 


\section{Acknowledgments}

This work was financially supported by National Natural Science Foundation of China (No. 61801169, No. 61573128, No. 61873086), the Jiangsu Province Natural Science Foundation (No. BK20170305) and the Fundamental Research Funds of Nanjing Hydraulic Research Institute (Y717010 and Y717009).

\section{References}

1. Jiang J.J., Duan F.J., Wang X.Q. 2D DOA and Frequency Estimation Method with One VectorSensor and Two Pressure Sensors Based on ESPRIT and Signal Power[J]. Wireless Personal Communications, 97(9):1-15(2017)

2. Pazos S, Hurtado M, Muravchik C. On Sparse Methods for Array Signal Processing in the Presence of Interference[J]. IEEE Antennas \& Wireless Propagation Letters, 14:1165-1168(2015)

3. Yan F.G., Jin T, Jin M, et al. Subspace-based direction-of-arrival estimation using centrosymmetrical arrays[J]. Electronics Letters, 52(22):1895-1896(2016)

4. Chen X.Q. Wang C.H. Zhang X.F.; DOA and Noncircular Phase Estimation of Noncircular Signal via an Improved Noncircular Rotational Invariance Propagator Method. Mathematical Problems in Engineering, 235173(2015)

5. Jiao Y.M., Huang J.G., Hou Y.S., Fastmaximumlikelihood direction-of-arrival estimator based on ant colony optimization. Systems Engineering and Electronics, 33, 1718-1721 (2011)

6. Boccato K., Krummenauer R., Attux R., Lopes A., Application of natural computing algorithms to maximum likelihood estimation of direction of arrival. Signal Processing, 92, 1338-1352(2012)

7. Zhang Z.C., Lin J., Shi Y.W., Application of artificial bee colony algorithm to maximum likelihood doa estimation. Journal of bionic engineering, 10, 100-109(2013)

8. Chen H.H., Li S.B., Liu J.H., Liu F., Suzuki M., A novel modification of PSO algorithm for SML estimation of DOA. Sensors, 16, 2188(2016)

9. Che C, Su J, Zheng X. Satellite Module Layout Design based on Adaptive Immune Bee Evolutionary Genetic Algorithm[J]. Journal of Convergence Information Technology(2014)

10. Kazimipour B., Li X.D., Qin A.K., A review of population initialization techniques for evolutionary algorithms. In 2014 IEEE Congress on Evolutionary Computation, 2585-2592(2014)

11. Qrito Y., Hanada Y., Shibata S., Yamamoto H., A new population initialization approach based on bordered hessian for portfolio optimization problems.
In 2013 IEEE International Conference on Systems, Man, and Cybernetics, 1341-1346(2013)

12. Bordel B., Orúe A.B., Alcarria R, et al. An intra-slice security solution for emerging $5 \mathrm{G}$ networks based on pseudo-random number generators[J]. IEEE Access, 6:16149-16164(2018) 